\title{
Entanglement entropy, trace anomalies and holography
}

\author{
A. Schwimmer ${ }^{a}$, S. Theisen ${ }^{b, *}$ \\ a Department of Physics of Complex Systems, Weizmann Institute, Rehovot 76100, Israel \\ b Max-Planck-Institut für Gravitationsphysik, Albert-Einstein-Institut, 14476 Golm, Germany
}

Received 25 February 2008; accepted 9 April 2008

Available online 22 April 2008

\begin{abstract}
The holographic representation of the entanglement entropy of four-dimensional conformal field theories is studied. By generalizing the replica trick the anomalous terms in the entanglement entropy are evaluated. The same terms in the holographic representation are calculated by a method which does not require the solution of the equations of motion or a cut-off. The two calculations disagree for rather generic geometries. The reasons for the disagreement are analyzed.
\end{abstract}

(c) 2008 Elsevier B.V. All rights reserved.

\section{Introduction}

Entanglement entropy [1] was proposed as a powerful tool for studying in detail the structure of vacua in QFT.

In $d=2$ CFT universal features of entanglement entropy (EE in the following) were studied in [2]: in particular the dependence of the EE on the scale of the region defining the EE was shown to be related to the trace anomaly of the CFT.

These results were generalized to any $d$ in [3]. Following the $d=2$ example [2] it was postulated that the EE in flat space is given by the usual partition function when the theory is defined in a metric with a conical singularity supported on a submanifold of codimension 2 . The submani-

\footnotetext{
Partially supported by the German-Israeli Project cooperation (DIP H52), the Einstein Center of the Weizmann Institute, the Humboldt Foundation and by the European Research and Training Networks 'Superstrings' (MRTN-CT2004-512194) (A.S.) and 'Forces Universe' (MRTN-CT-2004-005104) (S.T.).

* Corresponding author.

E-mail address: theisen@aei.mpg.de (S. Theisen).
} 
fold is the boundary between the two regions, $A$ and $B$, defining the EE. Universal terms in the scale dependence of the EE were again estimated by relating them to the trace anomalies (when $d$ is even) of the CFT. A very interesting proposal was made in [3] for a holographic realization of the EE: if the theory in flat space has a holographic dual $A d S_{d+1} \times X$ then the EE is realized by the above bulk gravitational theory to which one adds a $(d-1)$ Dirac-Nambu-Goto action (DNG action in the following) in the $A d S$ background, the coordinates at the boundary of $A d S$ representing the embedding of the $(d-2)$-dimensional boundary manifold.

This prescription allows in principle the calculation of all the observables in the EE at strong coupling including the complete scale dependence.

The prescription presupposes, as we will discuss in detail, that the theory in the singular metric can be represented equivalently by a smooth bulk metric to which an additional term defined on the singular manifold is added. Arguments for the validity of this assumption were presented in [4]. Using the aforementioned holographic representation for massive $d=4$ theories in [5] information about the phase structure of these theories was obtained; see also [6] for an earlier discussion of this issue.

In the present paper we reexamine the validity of the holographic prescription for the fourdimensional EE. We concentrate on the terms in the $d=4$ effective action representing the trace anomalies. These terms, being universal, can be controlled in a general CFT. Following the "replica trick" we deduce a singular metric which represents the EE. We assume that at least for the terms controlling the trace anomalies one can use the singular metric in the effective action without further regularization. We also assume that the "replica trick" expressions can be safely expanded to first order.

We conclude that for very generic cases (which include e.g. the situation when the boundary of the region defining the $\mathrm{EE}$ is a two-sphere) one cannot represent the singular metric by an additional piece localized on the singular submanifold. From a detailed study of the term in the effective action which generates the type A (Euler) trace anomaly we find that if the term is split into a $d=4$ part in a smooth metric and a $d=2$ part localized on the singular manifold the $d=2$ part does not have correct analyticity properties. If the splitting is done after a Weyl variation is taken (i.e. for the anomaly itself) the $d=2$ part does not fulfill the Wess-Zumino conditions. Since, as we will discuss in detail the holographic realization mentioned above has anomalous terms (bulk and Graham-Witten anomalies) with unambiguous "normal" analyticity properties it follows that the holographic prescription is not valid, at least for this particular, universal piece of the effective action. The analyticity requires that certain Bianchi identities are satisfied but the identities require contributions from singular and regular terms, preventing a consistent "splitting". The discrepancy between the CFT and its proposed holographic realization appears whenever the second fundamental form of the submanifold is nonvanishing.

One possibility we examine for explaining the discrepancy is to include the back reaction of the DNG action on the bulk component. It turns out that the back reaction does not change the anomalous terms.

Therefore another option for a holographic realization of the EE is the usual five-dimensional bulk action where at the boundary one matches to the singular four-dimensional metric. This prescription can be implemented at least for the anomaly calculations since, as we will discuss in detail, in this case one does not need to solve the classical equations of motion the anomalies being given by a direct evaluation of certain boundary terms. The validity of this straightforward prescription for other terms representing the EE as well as the origin of nonuniversal contributions to the EE requires further study. 
The holographic prescription containing the DNG action has the correct analytic structure to represent genuine extended observables in the CFT as it was shown explicitly in [7,8] and [9].

The paper is organized as follows: In Section 2 we generalize the replica trick of [2] to $d=4$ and we deduce an explicit form for the singular metric in the most general case needed for EE.

In Section 3 we discuss in detail the calculation of trace anomalies in the holographic set up both for the ones originating in the bulk and from the DNG piece of the action ("Graham-Witten anomalies"). We show that the calculation of the anomalies does not use the solution of the equations of motion reducing to the evaluation of a total derivative on the boundary. Furthermore this evaluation does not require the use of any cut-off procedure. Therefore the analyticity properties of the terms of the effective action responsible for the anomalies can be generally obtained.

In Section 4 we discuss the structure of the anomalous terms in the $d=4$ CFT in the singular metric representing the EE. We show that there is a contradiction between the structure of the type A (Euler) term and the one expected from the holographic description discussed in Section 3. We discuss the general mechanism which prevents the "splitting" of the EE problem into a bulk part and an additional piece formulated on the singular submanifold.

In Section 5 we study the influence of including the back reaction of the DNG action on the bulk. We show by a general argument that the back reaction does not change the anomalous terms. The argument is verified by solving the coupled equations of motion to the necessary order.

In Section 6 we calculate explicitly universal pieces of the EE for a sphere and we show that the results extracted from the CFT following our procedure disagree with the holographic prescription. In the same section we discuss open problems related to terms not controlled by the trace anomalies.

A new proof of a universal relation for the type A bulk trace anomaly [10] using the approach of Section 3 is presented in Appendix A.

\section{2. $\mathrm{EE}$ in $d=4$}

We start by discussing the four-dimensional generalization of the replica trick [2] we are going to use.

Consider a conformal field $\phi(\vec{x}, t)$ in $\mathbb{R}^{3,1}$. To simplify the notation we will consider a scalar field but our arguments should be valid for any spin. Let $A$ be a region in $\mathbb{R}^{3}$ and $B$ its complement. ${ }^{1}$ The boundary between $A$ and $B$ will be denoted $\partial$. We will start by assuming that the metric of space-time is flat but will later generalize it to a situation where the metric components can be space dependent such that the EE can still be defined.

The density matrix of the system has the path integral representation

$$
\begin{aligned}
& (\rho)_{\phi^{\prime}(\vec{x}, 0), \phi^{\prime \prime}(\vec{x}, 0)} \\
& =\frac{1}{Z} \int \mathcal{D} \phi(x) \prod_{x} \delta\left(\phi\left(\vec{x}, 0^{-}\right)-\phi^{\prime}(\vec{x}, 0)\right) \delta\left(\phi\left(\vec{x}, 0^{+}\right)-\phi^{\prime \prime}(\vec{x}, 0)\right) e^{-S[\phi]} .
\end{aligned}
$$

The reduced density matrix is obtained by setting $\phi^{\prime}(\vec{x}, 0)=\phi^{\prime \prime}(\vec{x}, 0)$ for $x \in B$ and integrating over $\phi$ which are continuous across $B$ :

\footnotetext{
1 For simplicity one might assume $A$ to be compact and connected.
} 


$$
\begin{aligned}
& \left(\rho_{A}\right)_{\phi^{\prime}(\vec{x}, 0), \phi^{\prime \prime}(\vec{x}, 0)} \\
& =\frac{1}{Z} \int \mathcal{D} \phi(x) \prod_{x \in A} \delta\left(\phi\left(\vec{x}, 0^{-}\right)-\phi^{\prime}(\vec{x}, 0)\right) \delta\left(\phi\left(\vec{x}, 0^{+}\right)-\phi^{\prime \prime}(\vec{x}, 0)\right) e^{-S[\phi]} .
\end{aligned}
$$

The entanglement entropy $S_{A}$ is the von Neumann entropy computed with the reduced density matrix $\rho_{A}$

$$
S_{A}=-\operatorname{Tr}_{A} \rho_{A} \log \rho_{A} .
$$

Various properties of the entanglement entropy are collected in [3].

The "standard" replica trick, by which

$$
S_{A}=-\lim _{n \rightarrow 1} \frac{\partial}{\partial n} \operatorname{Tr} \rho_{A}^{n}
$$

leads to $n$ copies $\phi^{(i)}$ of the field $\phi$ linked by the boundary conditions

$$
\phi_{A}^{(i)}\left(\vec{x}, t=0^{+}\right)=\phi_{A}^{(i+1)}\left(\vec{x}, t=0^{-}\right)
$$

where $\phi_{A}$ means that $\vec{x} \in A$ and $n+1 \equiv 1$. In addition in region $B$ the fields are continuous, i.e. single valued at $t=0$ :

$$
\phi_{B}^{(i)}\left(\vec{x}, t=0^{+}\right)=\phi_{B}^{(i)}\left(\vec{x}, t=0^{-}\right)
$$

where $\phi_{B}$ means $\vec{x} \in B$.

The action appearing in the path integral is

$$
S=\sum_{i=1}^{n} S\left[\phi_{A}^{(i)}, \phi_{B}^{(i)}\right]
$$

with the gluing specified in (2.5), (2.6). It is important that in the path integral there are no singularities: the time derivatives around $t=0$ are for $A$ :

$$
\left(\frac{\partial \phi}{\partial t}\right)^{2} \rightarrow\left(\frac{\phi^{(1)}(t+\epsilon)-\phi^{(2)}(t-\epsilon)}{2 \epsilon}\right)^{2}, \quad \text { etc. }
$$

Now define a new set of fields $\tilde{\phi}$ :

$$
\begin{aligned}
& \tilde{\phi}_{A}^{(i)}(\vec{x}, t)= \begin{cases}\phi_{A}^{(i)}(\vec{x}, t), & t>0, \\
\phi_{A}^{(i+1)}(\vec{x}, t), & t<0,\end{cases} \\
& \tilde{\phi}_{B}^{(i)}(\vec{x}, t)= \begin{cases}\phi_{B}^{(i)}(\vec{x}, t), & t>0, \\
\phi_{B}^{(i)}(\vec{x}, t), & t<0 .\end{cases}
\end{aligned}
$$

The fields $\tilde{\phi}^{(j)}$ are single valued at $t=0$ by construction.

However there is a new "gluing" condition due to the fact that $\phi^{(i)}$ was single valued at the boundary $\partial$ between $A$ and $B$, i.e.

$$
\left.\phi_{A}^{(i)}(\vec{x}, t)\right|_{\vec{x} \in \partial}=\left.\phi_{B}^{(i)}(\vec{x}, t)\right|_{\vec{x} \in \partial} .
$$

With the rearrangement (2.9), (2.10) becomes

$$
\left.\tilde{\phi}_{A}^{(i)}(\vec{x}, t)\right|_{\vec{x} \in \partial}=\left.\tilde{\phi}_{B}^{(i+1)}(\vec{x}, t)\right|_{\vec{x} \in \partial} \text { for } t<0 .
$$




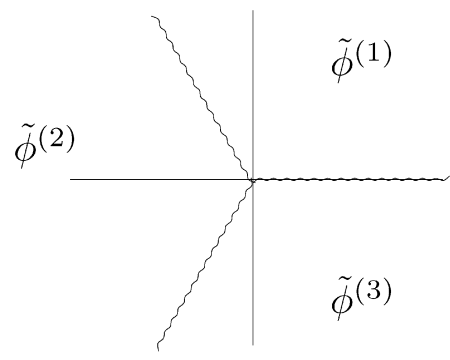

Fig. 1.

So the new formulation is: a set of $n$ replicas $\tilde{\phi}^{(i)}$ with a total action on single valued fields

$$
S=\sum_{i} S\left[\tilde{\phi}^{(i)}\right]
$$

and the gluing of different fields (2.11). The boundary conditions at $t= \pm \infty$ are left free for each $\tilde{\phi}^{(j)}$.

Following [2] we will now 'uniformize' the gluing manifold. The CFT is defined on $\mathbb{R}^{3,1}$ with the flat euclidean metric $\left(x^{4}=t\right)$

$$
d s^{2}=\left(d x^{1}\right)^{2}+\left(d x^{2}\right)^{2}+\left(d x^{3}\right)^{2}+\left(d x^{4}\right)^{2} .
$$

In the vicinity of the boundary $\partial$ we choose Gaussian normal coordinates $\left.\left(x^{i}\right)\right|_{i=1,2,3} \rightarrow\left(r, y^{a}\right)$, $a=1,2$, such that the boundary is located at $r=0$ and the metric is of the form

$$
d s^{2}=d t^{2}+d r^{2}+g_{a b}(r, x) d y^{a} d y^{b} .
$$

The induced metric on the $r=0$ hypersurface (at constant $t$ ) is $h_{a b}=\left.g_{a b}\right|_{r=0}$. The components of the second fundamental form $K_{a b}^{r}=-\left.\frac{1}{2} \partial_{r} g_{a b}\right|_{r=0}$ are generically non-zero. We remark that the choice of Gaussian coordinates leading to the metric (2.14) is also possible if we replace $\mathbb{R}^{3,1}$ by $\mathcal{M} \times \mathbb{R}$, where $\mathcal{M}$ is any three-manifold. The induced metric and second fundamental form are still expressed in terms of $g_{a b}$ as above.

The gluing manifold is now

$$
r=0, \quad t<0 .
$$

A further (singular) change of variables

$$
w \equiv i t+r=z^{n}
$$

brings all the gluing regions to the $z$ plane times $\partial$. This is exemplified for $n=3$ in Fig. 1 which shows the $z$-plane.

The metric becomes:

$$
d s^{2}=n^{2}(z \bar{z})^{(n-1)} d z d \bar{z}+g_{a b}(r, x) d y^{a} d y^{b} .
$$

For $n \neq 1$ this metric is singular on the hypersurface $z=0$ which is the boundary between the regions $A$ and $B$ at time $t=0$. This singularity is a consequence of the singularity of the coordinate transformation (2.16).

Since we are going to study what is happening when the metric undergoes a space-dependent Weyl transformation we allow (2.17) to be multiplied by such a factor such that finally the most 
general class of metrics we are studying is

$$
d s^{2}=2 g_{z \bar{z}}(r, x)(z \bar{z})^{(n-1)} d z d \bar{z}+g_{a b}(r, x) d y^{a} d y^{b}
$$

where $g_{z \bar{z}}$ is proportional to the respective metric component before the singular diffeomorphism.

\section{Holographic anomalies}

In this section we present a general scheme to compute holographic conformal anomalies. It is very much like the computation of the $S U(4) \mathcal{R}$-current anomaly presented in [11]. The anomaly is the boundary term generated by a suitably chosen local symmetry transformation. In the case of the $\mathcal{R}$-current this is a $S U(4)$ gauge transformation and the boundary term, which is the $\mathcal{R}$-current anomaly of the CFT on the boundary $(\mathcal{N}=4$ SYM) is due to the $S U$ (4) ChernSimons term present in the $5 \mathrm{~d}$ gauged supergravity that arises when one compactifies type IIB string theory on $S^{5}$. For the conformal anomalies the appropriate transformations are the socalled PBH-transformations, a subgroup of five-dimensional diffeomorphisms introduced in [10] and reviewed in the following. This treatment of the conformal anomalies does not require the solution of the equations of motion and it does not depend on the introduction of a cut-off. This gives us confidence on the generality of their structure when we compare it with the results in the field theory.

In the first part of this section we deal with those anomalies origination from the bulk gravitational action. In the second part we extend the discussion to the trace anomalies originating from the DNG piece of the action ("Graham-Witten anomalies").

\subsection{Anomalies from the bulk}

We start with trace anomalies in the bulk. Besides giving a general illustration of the new way to calculate trace anomalies the explicit results will be used in the following for an alternative holographic representation of the anomalous pieces in the EE.

Consider a generic gravitational bulk action

$$
S=\int_{M} \sqrt{G} f(R) d^{d+1} X
$$

where $f$ is an arbitrary scalar function of the curvature and its derivatives. We require that (3.1) admits $A d S_{d+1}$ as a solution to the equations of motion: this imposes a mild inequality on the coefficients in $f(R)$.

We choose coordinates $X^{\mu}=\left(x^{i}, \rho\right)$ such that $\rho=0$ is the boundary of $A d S_{d+1}$ where the dual CFT lives. It is coupled to a metric (source for its energy momentum tensor) $g_{i j}^{(0)}(x)$. For the bulk metric we choose the Fefferman-Graham (FG) gauge [12,13]

$$
d s^{2}=G_{\mu \nu} d X^{\mu} d X^{\nu}=\left(\frac{d \rho}{2 \rho}\right)^{2}+\frac{1}{\rho} g_{i j}(x, \rho) d x^{i} d x^{j}
$$

with $g_{i j}(x, 0)=g_{i j}^{(0)}(x)$. For $g_{i j}^{(0)}=\eta_{i j}$ (3.2) is the metric of $A d S_{d+1}$, whose curvature radius we have set to one.

PBH (Penrose-Brown-Henneaux) transformations are those diffeomorphisms $\xi^{\mu}$ which preserve the FG-gauge [10], i.e. for which $\mathcal{L}_{\xi} G_{\rho \rho}=\mathcal{L}_{\xi} G_{\rho i}=0$. The solution is parametrized by an 
arbitrary function $\sigma(x)^{2}$ :

$$
\xi^{\rho}=-2 \rho \sigma(x), \quad \xi^{i}=a^{i}(x, \rho)=\frac{1}{2} \int_{0}^{\rho} d \rho^{\prime} g^{i j}\left(x, \rho^{\prime}\right) \partial_{j} \sigma(x) .
$$

In particular, $\delta_{\sigma} g_{i j}^{(0)}=2 \sigma g_{i j}^{(0)}$, i.e. $\sigma(x)$ is the parameter of Weyl rescalings of the boundary metric.

The group property for $\mathrm{PBH}$ transformations can be shown to be

$$
\xi_{1}^{\nu} \partial_{\nu} \xi_{2}^{\mu}-\xi_{2}^{\nu} \partial_{\nu} \xi_{1}^{\mu}+\delta_{2} \xi_{1}^{\mu}-\delta_{1} \xi_{2}^{\mu}=0 .
$$

The last two terms are due to the dependence of the transformation parameters on $g_{i j}(x, \rho)$.

The essential property of the PBH transformations is that on the boundary they coincide with the action of the Weyl group. Therefore in holography the Weyl group becomes embedded in the $(d+1)$-dimensional diffeomorphisms and the study of Weyl anomalies is reduced to an analysis of how diffeomorphisms act.

Under a bulk diffeomorphism the action (3.1) is invariant up to a boundary term

$$
\begin{aligned}
& \delta_{\xi}(\sqrt{G} f)=\partial_{\mu}\left(\sqrt{G} \xi^{\mu} f\right), \\
& \delta_{\xi} S=\left.\int_{\partial M} d^{d} x \sqrt{G} f(R) \xi^{\rho}\right|_{\rho=0}=-\left.2 \int_{\partial M} d^{d} x \sqrt{G} f(R) \rho \sigma\right|_{\rho=0},
\end{aligned}
$$

where in the second line we have restricted the diffeomorphism to a PBH transformation. The finite piece of this boundary term is the holographic Weyl anomaly.

A comment is in order here: we consider passive diffeomorphism transformations which act on the fields rather than the coordinates. The reason for doing is that we want to keep the boundary fixed.

Following [12] (see also [14]) we expand the metric as

$$
g_{i j}(x, \rho)=\sum_{n=0}^{\infty} \stackrel{(n)}{g}_{i j}(x) \rho^{n}+\cdots
$$

The $\cdots$ denote logarithmic terms $(\sim \log \rho)$ which are present for even $d$. They do not play a role in our analysis. The integrand in (3.1) has likewise an expansion of the form [10]

$$
\sqrt{G} f(R)=\sqrt{g^{(0)}} \rho^{-\frac{d}{2}-1} b(x, \rho)=\sqrt{g^{(0)}} \rho^{-\frac{d}{2}-1} \sum_{n=0}^{\infty} b_{n}(x) \rho^{n} .
$$

As was shown in [10], $b$ and thus each $b_{n}$, satisfies the Wess-Zumino consistency condition

$$
\int d^{d} x \sqrt{g^{(0)}}\left(\sigma_{1} \delta_{\sigma_{2}}-\sigma_{2} \delta_{\sigma_{1}}\right) b=0
$$

A simple way to see this is as follows (cf. Appendix A). For $\mathcal{O}=\sqrt{G} f(R)$ one derives $\delta_{\sigma_{1}} \mathcal{O}=$ $\partial_{\mu}\left(\xi_{1}^{\mu} \mathcal{O}\right)$ and $\left[\delta_{\sigma_{2}}, \delta_{\sigma_{1}}\right] \mathcal{O}=0$ by virtue of the group property (3.4).

\footnotetext{
2 The choice of lower limit in the $\rho^{\prime}$ integral means that we do not consider diffeomorphisms of the boundary. They are of no interest here.
} 
On-shell $b_{n}$ is a local, covariant expression constructed from $g_{i j}^{(0)}$. For $d=2 n$ it is the coefficient of the boundary term at $\mathcal{O}\left(\rho^{-1}\right)$ and represents the Weyl anomaly of the dual $2 n$ dimensional CFT. The bulk gravitational action thus plays the same role for the Weyl anomaly of the CFT as does the CS term for the $\mathcal{R}$-current anomaly.

For general $d=2 n$, the on-shell $b_{n}$ depends also on some of the derivatives of $g_{i j}$ at $\rho=0$ and not only on the boundary value $g_{i j}^{(0)}$. These higher derivatives need some information contained in the equation of motion. However, for $d=4, b_{2}$ can be computed without the need to solve the equations of motion. As we will show momentarily, in $d=4$, besides $g^{(0)}$ only the coefficient $g^{(1)}$ of the FG expansion of the bulk metric appears. This second coefficient is universal because it is uniquely determined by its behavior under PBH transformations and locality [10]:

$$
\stackrel{(1)}{g}_{i j}=\frac{1}{(d-2)}\left(R_{i j}-\frac{1}{2(d-1)} g_{i j} R\right),
$$

where $R$ is the curvature of $g^{(0)}$ and $g_{i j} \equiv g_{i j}^{(0)}$. The universality of $g^{(1)}$ will be spoiled if we take back reaction into account, as we will do in Section 5.

On dimensional grounds $b_{n}$ can at most be linear in $g^{(n)}$, as both carry length-dimension $-2 n$ $\left(\rho \sim\right.$ length $\left.^{2}\right)$. By assumption, $f(R)$ is such that anti-de Sitter space is a solution of the equations of motion. Expand the action around this solution. In this expansion the term linear in the fluctuations around the $A d S$-metric can only be a total derivative (or vanish altogether). Consider the terms $\nabla^{\mu} \nabla^{v} \delta G_{\mu \nu}$ and $\square \operatorname{tr} \delta G$. For fluctuations $\delta G_{i j}=\rho^{n-1} g_{i j}^{(n)}$ the possibly dangerous terms, i.e. those which might contribute to $b_{n}$, are of the type $\rho^{n} \operatorname{tr} g^{(n)}$. It is straightforward to show that their coefficient is zero for $d=2 n$. Higher derivative terms in the variation of the action will involve coefficients $g^{(m)}$ for $m<n$. We stress that the above argument showing that in $d=2 n g^{(n)}$ does not appear in the $b_{n}$ term in the expansion of the action does not prevent the participation of $g^{(n)}$ in the equation of motion in the usual way [13] of calculating the anomalies. ${ }^{3}$

To summarize, to find the Weyl anomaly of the $d=2 n$-dimensional dual CFT all we have to do is to extract the coefficient of $1 / \rho$ is the expansion of the gravitational action. In $d=4$ this only involves $g^{(0)}$ and $g^{(1)}$ and is thus completely fixed. On general grounds this can always be written as a linear combination $a E_{4}-c C^{2}+e \square R$ where $C^{2}$ is the square of the Weyl tensor, $E_{4}$ the Euler density (i.e. $\int_{M} \sqrt{g} E_{4} \propto \chi(M)$ ). In Appendix A we will rederive the general expression for $a$, already found, by different means, in [10].

\subsection{Graham-Witten anomalies}

In this subsection we will study the trace anomalies for submanifolds ("Graham-Witten" anomalies) which are of direct relevance for the proposed holographic dual of EE. We will follow the method used in the previous subsection for bulk anomalies which does not depend on the equations of motion and does not need a cut-off. This will enable us to discuss the general structure of the Graham-Witten anomalies needed for EE and the anomalies produced by more general submanifold actions having the same symmetries as DNG. For the DNG action our method reproduces the result of [17].

We start with a classification of the possible Graham-Witten anomalies for the case when the submanifold has dimension 2 embedded in a manifold of dimension $d$.

\footnotetext{
3 Alternative arguments for obtaining anomalies without solving the equations of motion were given in $[15,16]$.
} 
Candidates for the Graham-Witten anomaly are solutions to the Wess-Zumino consistency condition satisfying the following conditions: they should be local expressions constructed from the second fundamental form and from curvatures, linear in the Weyl parameter $\sigma$; they should have two derivatives (appropriate for the two-dimensional case considered here); they should be cohomologically non-trivial. Among those we distinguish between type A which satisfy the WZ condition non-trivially and type B which satisfy them trivially having expressions which are Weyl invariant.

To find the candidates for the anomaly, we will need, besides well-known expressions for the Weyl-transformation of the curvature tensors, the transformation of the second fundamental form and of its trace (cf. below):

$$
\delta_{\sigma} K_{a b}^{i}=-h_{a b} P^{i j} \partial_{j} \sigma, \quad \delta_{\sigma} K^{i} \equiv \delta_{\sigma}\left(h^{a b} K_{a b}^{i}\right)=-2 \sigma K^{i}-k P^{i j} \partial_{j} \sigma,
$$

where $P^{i j}=g^{i j}-h^{i j}=g^{i j}-h^{a b} \partial_{a} X^{i} \partial_{b} X^{j}$ projects to the normal space of the hypersurface. The derivatives $\partial_{a}$ are with respect to the local coordinates on the submanifold and $X^{i}$ are the embedding functions.

It is then straightforward to show that the following list exhausts all possible Weyl invariant expressions:

$$
\begin{aligned}
& \sqrt{h} h^{a c} h^{b d} C_{a b c d}, \quad \sqrt{h}\left(\operatorname{tr}\left(K^{i} K^{j}\right)-\frac{1}{2} K^{i} K^{j}\right) g_{i j}, \\
& \sqrt{h}\left(K^{i} K^{j} g_{i j}-4 h^{a b_{g}^{(1)}}+2 R^{(2)}\right),
\end{aligned}
$$

where $R^{(2)}$ is the curvature scalar of the induced metric, $C_{a b c d}$ the pull-back of the bulk Weyl tensor and $\stackrel{(1)}{g}_{a b}=\partial_{a} \stackrel{(0)}{X}^{i} \partial_{b} \stackrel{(0)}{X}^{j} \stackrel{(1)}{g}_{i j}$ is the pull-back of (3.10). However, with the help of the GaussCodazzi equation one shows that

$$
h^{a c} h^{b d} C_{a b c d}=R^{(2)}-2 h^{a b^{(1)}}{ }_{a b}+\frac{1}{2} K^{i} K^{j} g_{i j}-\left(\operatorname{tr}\left(K^{i} K^{j}\right)-\frac{1}{2} K^{i} K^{j}\right) g_{i j},
$$

i.e. the above Weyl invariant expressions are not all independent. We will choose the first two as a basis.

Candidates for the type A anomaly are

$$
\sqrt{h} R^{(2)} \sigma, \quad \sqrt{h} K^{i} \partial_{i} \sigma, \quad \sqrt{h} \square \sigma,
$$

where these expressions are restricted to the submanifold. The first is the well-known trace anomaly in $d=2$. The second one, on the other hand, is trivial as it can be written as the Weyl variation of a local term:

$$
\delta_{\sigma}\left(K^{i} K^{j} g_{i j}\right)=-4 K^{i} \partial_{i} \sigma
$$

where one uses (3.11) and $K_{i} h^{i j}=0$. The third one is again trivial being the variation of $R$ the scalar bulk curvature restricted to the submanifold.

We thus arrive at the following basis of GW anomalies when the submanifold is twodimensional:

type A: $\quad \sqrt{h} R^{(2)} \sigma$,

type B: $\quad \sqrt{h} h^{a c} h^{b d} C_{a b c d} \sigma, \quad \sqrt{h} g_{i j}\left(\operatorname{tr}\left(K^{i} K^{j}\right)-\frac{1}{2} K^{i} K^{j}\right) \sigma$. 
In terms of this basis the anomaly found by Graham and Witten, who considered the case where the hypersurface degrees of freedom in the CFT have their holographic description in terms of the DNG action, is

$$
\mathcal{A}_{\mathrm{GW}}=\frac{1}{4} \int_{\partial \Sigma} d^{2} x \sqrt{h}\left(h^{a c} h^{b d} C_{a b c d}-g_{i j}\left(\operatorname{tr}\left(K^{i} K^{j}\right)-\frac{1}{2} K^{i} K^{j}\right)-R^{(2)}\right) \sigma .
$$

We proceed now to an analysis of the Graham-Witten anomalies in a holographic setup. We will leave the dimensions of space-time $d$ and of the submanifold $k$ general and at the end of the discussion we will go back to the specific $k=2$ case.

In the holographic realization we have to consider a $(k+1)$-dimensional submanifold $\Sigma$ embedded into the $(d+1)$-dimensional bulk $M$ such that it ends on a $k$-dimensional submanifold ว $\Sigma$ on the $d$-dimensional boundary. Denote, as before, the bulk coordinates by $X^{\mu}=\left(x^{i}, \rho\right)$ and the world-volume coordinates by $\tau^{\alpha}=\left(y^{a}, \tau\right)$ with $i=1, \ldots, d$ and $a=1, \ldots, k$. The embedding is $X^{\mu}: \Sigma \mapsto M$, i.e. $X^{\mu}=X^{\mu}\left(\tau^{\alpha}\right)$.

We assume that the action contains in addition to the usual bulk component (3.1) another component defined on the $k+1$ submanifold. The additional piece is invariant both under usual bulk diffeomorphisms and under reparametrizations of the world volume.

We want first to generalize the PBH transformations (3.3) to this new situation where we have two linked gauge invariances.

We first fix the gauge. For the bulk we go to FG gauge (3.2) as before. The reparametrizations of $\Sigma$ are fixed by imposing

$$
\tau=\rho \quad \text { and } \quad h_{a \tau}=0 .
$$

Under a reparametrization of $\Sigma$, parametrized by $\tilde{\xi}^{\alpha}, X^{\mu}$ transforms as a scalar, i.e. $\delta_{\tilde{\xi}} X^{\mu}=$ $\tilde{\xi}^{\alpha} \partial_{\alpha} X^{\mu}$. In particular $\delta_{\tilde{\xi}} \rho=\tilde{\xi}^{\alpha} \partial_{\alpha} \rho=\tilde{\xi}^{\tau}=0$ after fixing the $\tau=\rho$ gauge. Also, if we require that $\delta_{\tilde{\xi}} h_{a \tau}=0$, we find that $\tilde{\xi}^{a}$ must be independent of $\tau$. This means that all world-volume reparametrizations of $\Sigma$ are fixed except the ones acting on $\partial \Sigma$.

We perform now a target space PBH transformations $\delta \rho=-2 \rho \sigma, \delta x^{i}=a^{i}$ (cf. (3.3)). To stay in the $\tau=\rho$ gauge we must make a compensating world-volume diffeomorphism

$$
\tilde{\xi}^{\tau}=-2 \tau \sigma .
$$

The resulting change of the induced metric must be compensated in order to keep $h_{a \tau}=0$ :

$$
\delta h_{a \tau}=\partial_{a} \tilde{\xi}^{\tau} h_{\tau \tau}+\partial_{\tau} \tilde{\xi}^{b} h_{a b}=0 .
$$

With $\tilde{\xi}^{\tau}=-2 \tau \sigma$ this can be integrated to

$$
\tilde{\xi}^{a}=2 \int_{0}^{\tau} d \tau^{\prime} \tau^{\prime} h_{\tau \tau} h^{a b} \partial_{b} \sigma
$$

where all functions in the integrand depend on $\tau^{\prime}$ (through $\left.X^{i}\left(y^{a}, \tau\right)\right)$. Here

$$
h_{\tau \tau}=\partial_{\tau} X^{\mu} \partial_{\tau} X^{\nu} G_{\mu \nu}=\frac{1}{4 \tau^{2}}+\frac{1}{\tau} \partial_{\tau} X^{i} \partial_{\tau} X^{j} g_{i j}(X, \tau) \text {. }
$$

Expand $g_{i j}$ in powers of $\rho$ (cf. (3.7)) and $X^{i}$ in powers of $\tau$ (with $\tau=\rho$ )

$$
X^{i}\left(\tau, y^{a}\right)=\stackrel{(0)}{X}^{i}\left(y^{a}\right)+\tau \stackrel{(1)}{X^{i}}\left(y^{a}\right)+\tau^{2} \stackrel{(2)}{X}^{i}\left(y^{a}\right)+\cdots .
$$


With the definition

$$
\begin{aligned}
h_{a b} & =\frac{1}{\rho} \partial_{a} X^{i} \partial_{b} X^{j} g_{i j}(X) \\
& =\frac{1}{\rho} \partial_{a} \stackrel{(0)}{X}^{i} \partial_{b} \stackrel{(0)}{X}^{j} \stackrel{(0)}{g}_{i j}(\stackrel{(0)}{X})+\mathcal{O}(1) \equiv \frac{1}{\rho} \stackrel{(0)}{h}_{a b}(X)+\mathcal{O}(1)
\end{aligned}
$$

we obtain from (3.21)

$$
\tilde{\xi}^{a}=\frac{1}{2} \tau h^{(0)} \partial_{b} \sigma+\mathcal{O}\left(\tau^{2}\right) .
$$

We can now determine how $X^{i}$ changes under PBH. It transforms as

$$
\delta X^{i}=\tilde{\xi}^{\alpha} \partial_{\alpha} X^{i}-a^{i}
$$

with $a^{i}$ from (3.3). This implies

$$
\begin{aligned}
& \delta \stackrel{(0)}{X}^{i}=0, \\
& \delta \stackrel{(1)}{X}^{i}=-2 \sigma \stackrel{(1)}{X}^{i}+\frac{1}{2} \stackrel{(0)}{h}^{a b} \partial_{a} \stackrel{(0)}{X}^{i} \partial_{b} \sigma-\frac{1}{2} \stackrel{(0)}{g}^{i j} \partial_{i} \sigma
\end{aligned}
$$

which is solved by

$$
\stackrel{(1)}{X}^{i}=\frac{1}{2 k} K^{i}
$$

where

$$
K^{i}=\stackrel{(0)}{h}^{a b} K_{a b}^{i}=\stackrel{(0)}{h}^{a b}\left(\partial_{a} \partial_{b} \stackrel{(0)}{X}^{i}-\stackrel{(0)}{\Gamma}_{a b}^{c} \partial_{c} \stackrel{(0)}{X}^{i}+\stackrel{(0)}{\Gamma}_{j k}^{i} \partial_{a} \stackrel{(0)}{X}^{j} \partial_{b} \stackrel{(0)}{X}^{k}\right)
$$

is the trace of the second fundamental form, i.e. the extrinsic curvature, of the embedded submanifold $\partial \Sigma$.

We remark that the universality of $X^{i}$ is analogous to the universality of $g^{(1)}$, cf. (3.10). The higher $X^{(n)}$, just like the higher $g^{(n)}$, are not universal, the reason being that their behavior under PBH transformations admits homogeneously transforming terms. ${ }^{4}$

We succeeded therefore to put also this more general situation, with the action having two components, into a framework similar to the one we had for the bulk action alone. The action of the Weyl transformations on the boundary is embedded into bulk diffeomorphisms and world volume reparametrizations (3.19), (3.21). Moreover, besides the $g^{(1)}$ component of the bulk metric also the $X^{(1)}$ component of the embedding have a universal form determined by the $\mathrm{PBH}$ transformations.

Using these results we are now prepared to analyze the Graham-Witten anomalies, i.e. the transformation properties of the additional piece of the action when the metric $g^{(0)}$ is Weyl transformed.

Following [17] we consider the case where the dynamics of the submanifold is governed by the DNG action:

$$
S=\int_{\Sigma} \sqrt{h} .
$$

${ }^{4}$ For $g_{i j}^{(2)}$ this is e.g. $g_{i j}^{(0)} C^{2}$ and for $X^{(1)}$ any one of the terms in (3.12) (without the $\sqrt{h}$ factor), multiplied by $X^{(0)}$. 
The generalization to arbitrary world-volume actions is straightforward. A particular case will be considered at the end of this section. The DNG action of $\Sigma$ is invariant under passive worldvolume diffeomorphisms up to a boundary term. The finite part of this boundary term $($ at $\tau=0)$ is the Graham-Witten anomaly

$$
\mathcal{A}=\left.\int_{\partial \Sigma} \sqrt{\operatorname{det} h} \tilde{\xi}^{\tau}\right|_{\text {finite }} .
$$

Given that the $\tau$-expansion of $X^{(1)}$ is universal only up to the first non-trivial order, we will be able to compute the anomaly, without further input from the equations of motion, only for $k=2$. This is also the relevant dimension for the discussion of the EE in a four-dimensional CFT.

We now evaluate (3.31). We need

$$
\tilde{\xi}^{\tau}=-2 \tau \sigma(X)=-2 \tau \sigma(\stackrel{(0)}{X})-2 \tau^{2} \partial_{i} \sigma(\stackrel{(0)}{X}) \stackrel{(1)}{X}^{i}+\mathcal{O}\left(\tau^{3}\right)
$$

and $\operatorname{det}(h)=h_{\tau \tau} \operatorname{det}\left(h_{a b}\right)$ with

$$
\begin{aligned}
& h_{\tau \tau}=\frac{1}{4 \tau^{2}}+\frac{1}{\tau} \stackrel{(1)}{X}^{i} \stackrel{(1)}{X}^{j} \stackrel{(0)}{g}_{i j}+\cdots=\frac{1}{4 \tau^{2}}\left(1+4 \tau \stackrel{(1)}{X^{i}} \stackrel{(1)}{X}^{j} \stackrel{(0)}{g}_{i j}\right)+\cdots, \\
& \operatorname{det} h_{a b}=\frac{1}{\tau^{k}} \operatorname{det}\left(\stackrel{(0)}{h}_{a b}\right)\left(1+\tau \stackrel{(0)}{h}^{a}{ }^{(1)} h_{a b}\right)+\cdots,
\end{aligned}
$$

where

$$
\begin{aligned}
& \stackrel{(1)}{h_{a b}}=\partial_{a} \stackrel{(1)}{X}^{i} \partial_{b} \stackrel{(0)}{X^{j}}{\stackrel{(0)}{g_{i j}}}_{i j}+\partial_{a} \stackrel{(0)}{X}^{i} \partial_{b} \stackrel{(1)}{X}^{j} \stackrel{(0)}{g}_{i j}+\partial_{a} \stackrel{(0)}{X}^{i} \partial_{b} \stackrel{(0)}{X}^{j} \stackrel{(1)}{g}_{i j}+\partial_{a} \stackrel{(0)}{X}^{i} \partial_{b} \stackrel{(0)}{X}^{j} \partial_{k} \stackrel{(0)}{g}_{i j} \stackrel{(1)}{X}^{k} \\
& =\stackrel{(1)}{g}_{a b}-\frac{1}{k} K^{i} K_{a b}^{j}{\stackrel{(0)}{g_{i j}}}_{i j} .
\end{aligned}
$$

With the help of these expressions we finally find, for $k=2$,

$$
\mathcal{A}_{\mathrm{GW}}=\frac{1}{8} \int_{\partial \Sigma} d^{2} y \sqrt{\operatorname{det} h}\left(\left(g_{i j} K^{i} K^{j}-4 h^{a b_{g}^{(1)}}\right) \sigma-2 K^{i} \partial_{i} \sigma\right),
$$

where $h$ and $g$ now denote the boundary metrics. Eq. (3.35) is in agreement with [17]. As remarked above, the last term is cohomologically trivial. The rest can be written in terms of the basis (3.16). The result was already given in (3.17).

In analogy to allowing general bulk actions, as we did in Section 3.1, the dynamics of the hypersurface might be given by generalizations of the DNG action

$$
S=\int_{\Sigma} \sqrt{h} f\left(R^{\Sigma}, K, X, \ldots\right)
$$

where $f$ is a scalar function. In this case the GW anomaly will also change:

$$
\mathcal{A}=\left.\int_{\partial \Sigma} \sqrt{\operatorname{det} h} f \tilde{\xi}^{\tau}\right|_{\text {finite }} .
$$

The fact that the anomaly satisfies the $\mathrm{WZ}$ condition is again a consequence of the group property of the PBH transformations.

As a particular example we consider the action

$$
S=\int_{\Sigma} \sqrt{h} f\left(R^{(\Sigma)}\right)
$$


where $R^{(\Sigma)}$ is the Ricci scalar computed with $h_{\alpha \beta}$ with the expansion

$$
R^{(\Sigma)}=6+\left(R^{(2)}-2 h^{a b^{(1)}}{ }_{a b}+\frac{1}{2} \stackrel{(0)}{g}_{i j} K^{i} K^{j}\right) \tau+\cdots .
$$

For instance, if we choose $f\left(R^{(\Sigma)}\right)=1-\frac{1}{2} R^{(\Sigma)}$ the GW-anomaly is purely type A. Alternatively we can choose an action for which the $R^{(2)}$ anomaly vanishes which could be relevant for the EE as we discuss in Section 5.

\section{Anomalies in the singular metric background}

We will study the CFT in the singular metric (2.18) obtained in Section 2 to which the CFT should be coupled in order to calculate the EE. The metric is the result of a singular diffeomorphism (2.16) applied to the original, smooth metric.

We will assume that in the generating functional the singular metric can be used for calculating the EE at least for the terms generating the trace anomalies without the need for additional regularization.

Since through the "replica trick" the EE requires only the derivative with respect to the replica number $n$ at $n=1$ we will expand everything in $\epsilon=n-1$ and keep only the first order term in $\epsilon$.

The thus expanded, singular diffeomorphism is given by:

$$
w=z+\epsilon z \log (z), \quad \bar{w}=\bar{z}+\epsilon \bar{z} \log (\bar{z}) .
$$

As a consequence of the diffeomorphism being singular there could be two-dimensional $\delta$-function contributions in certain curvature components. The contribution which can give $\delta$-function appears in $g_{z \bar{z}}$ :

$$
\epsilon g_{z \bar{z}} \log (z \bar{z}) \text {. }
$$

Using

$$
\partial_{z} \partial_{\bar{z}} \log (z \bar{z})=4 \pi \delta^{(2)}(z, \bar{z})
$$

the singular contribution is:

$$
\bar{R}_{z \bar{z} z \bar{z}}=-4 \pi \epsilon g_{z \bar{z}} \delta^{(2)}(z, \bar{z})
$$

where we denote with $\bar{R}$... the contributions to the transformed curvature components which have a $\delta$-function. In addition the transformed components will have contributions denoted by $\tilde{R}$... through the action of (4.1) treated as a regular diffeomorphism on the original components $R \ldots$

We list the components of the Ricci tensor and the scalar curvature which are singular as a consequence of (4.4):

$$
\bar{R}_{z \bar{z}}=4 \pi \epsilon \delta^{(2)}(z, \bar{z}), \quad \bar{R}=8 \pi \epsilon g^{z \bar{z}} \delta^{(2)}(z, \bar{z}) .
$$

It is tempting to separate the "regular" and "singular" pieces of the curvatures representing the EE to give respectively a four-dimensional theory in a smooth metric and an effectively twodimensional contribution obtained from the singular piece after integrating the $\delta$-function. Such a separation would justify the holographic representation proposed in [3] where in addition to the five-dimensional bulk theory there is the DNG action representing the two-dimensional, singular contribution. 
However, as discussed in Section 3 the aforementioned holographic setup leads to a well defined, specific analyticity structure. In order that the holographic mapping makes sense the same analyticity structure should exist in the original theory. In particular the holographic representation leads to an effective action depending on the boundary variables (metric and embedding functions of the submanifold) which is Weyl invariant up to local anomalies. This means that:

(a) under a Weyl transformation the effective action is invariant except for local terms: fourdimensional ("the bulk trace anomalies") and two-dimensional ("the Graham-Witten anomalies") and

(b) the anomalies fulfill the Wess-Zumino condition, i.e. a further Weyl variation antisymmetrized with the first one should vanish.

We will study the above conditions for the universal pieces of the effective action of the EE field theory responsible for the trace anomalies and show that they fail for rather generic geometries. We believe that similar failures probably occur also in other terms of the effective action which however are specific to the various CFT.

The calculation is rather straightforward. We introduce the singular metric to first order in $\epsilon$ into the effective action and the Weyl anomalies obtained from it by making a Weyl variation. The expressions make sense to first order in $\epsilon$ in the four-dimensional sense. We will try, however, to "split" the expressions into a four-dimensional piece corresponding to the regular part of the curvatures and a two-dimensional piece contributed by the components of the curvatures which have an explicit $\delta$-function and we will check if the two pieces have the properties of the holographic representation.

If the splitting is done at the level of the effective action this requires that after a Weyl variation the two pieces produce local Weyl anomalies, bulk (four-dimensional) and Graham-Witten (twodimensional), respectively.

If the splitting is done for the Weyl anomalies in the background of the singular metric (again to first order in $\epsilon$ ) the two pieces should obey the Wess-Zumino conditions in four and two dimensions, respectively.

Both "splittings" fail for the part of the effective action producing the type A (Euler) anomaly if the embedding geometry has a nonvanishing second fundamental form. An analysis of the trace anomalies for the EE when the second fundamental form vanishes was performed in [3] using the geometric setup discussed in [18].

Since the analysis of the nonlocal anomalous pieces of the action is somehow cumbersome we will use the Wess-Zumino actions [19] in which only manipulation of local terms is needed the results being completely equivalent. The Wess-Zumino actions replace the nonlocality with the introduction of another scalar field $\phi\left(x^{i}\right)$, the parameter field of the Weyl group. The dependence of the action on the metric $g_{i j}$ and on $\phi$ is local. The Weyl transformation of the fields is:

$$
g_{i j}^{\prime}\left(x^{k}\right)=\exp \left(2 \sigma\left(x^{\kappa}\right)\right) g_{i j}\left(x^{k}\right), \quad \phi^{\prime}\left(x^{k}\right)=\phi\left(x^{k}\right)+\sigma\left(x^{k}\right),
$$

where $\sigma\left(x^{k}\right)$ is the parameter of the Weyl transformation. We require that the effective action $W\left(g_{i j}, \phi\right)$ under the transformation (4.6) produces the anomalies, i.e.

$$
\delta_{\sigma} W=\int d^{d} x \sigma \mathcal{A}
$$


where the anomaly $\mathcal{A}$ depends only on $g_{i j}$. The effective actions $W$ are obtained by a general procedure [19] and we reproduce here their form in $d=2$ and $d=4$ we will need:

$$
W_{2}=a_{2} \int d^{2} x \sqrt{\operatorname{det}(g)}\left[\phi R+g^{a b} \partial_{a} \phi \partial_{b} \phi\right]
$$

where $a_{2}$ is the unique two-dimensional trace anomaly coefficient, and [20]

$$
\begin{aligned}
W_{4}= & \int d^{4} x \sqrt{\operatorname{det}(g)}\left(a _ { 4 } \left[\phi E_{(4)}-4 G_{i j} \partial^{i} \phi \partial^{j} \phi+2\left(\partial_{i} \phi \partial^{i} \phi\right)^{2}\right.\right. \\
& \left.\left.-4 \square \phi \partial_{i} \phi \partial^{i} \phi\right]+c_{4} \phi C_{(4)}^{2}\right),
\end{aligned}
$$

where $E_{(4)}$ is the four-dimensional Euler density, $C_{(4)}^{2}$ is the square of the four-dimensional Weyl tensor, $G_{i j}$ is the Einstein tensor

$$
G_{i j}=R_{i j}-\frac{1}{2} g_{i j} R
$$

and $a_{4}$ and $c_{4}$ are the coefficients of the two four-dimensional trace anomalies.

To the expressions (4.8), (4.9) we can add arbitrary terms made of $\phi$ and $g$ which are Weyl invariant, in particular the quadratic action of a conformally coupled scalar $\Phi$, the exponential of $\phi$.

If we express $\phi$ through the equation of motions in terms of $g$ and use it in (4.8) or (4.9) Eq. (4.7) is still fulfilled, i.e. we obtain the nonlocal action generating the anomaly. We illustrate this procedure in $d=2$ : The $\phi$ equation of motion gives:

$$
\phi=\frac{1}{2 \square} R
$$

and using (4.11) in (4.8) we obtain:

$$
W_{2}=\frac{a_{2}}{4} \int d^{2} x \sqrt{\operatorname{det}(g)} R \frac{1}{\square} R,
$$

i.e. the Polyakov action.

A similar procedure can be done in $d=4$ solving the equation of motion following from (4.9) as an expansion in powers of $\phi$. We will not need the explicit form of the nonlocal action obtained this way being more convenient to work with the local action (4.9) containing $\phi$.

We will try to implement the "splitting" mentioned above by using the singular and regular components of the Riemann tensor in (4.9). The terms which could have $\delta$-functions are the ones containing $E_{(4)}, G_{i j}$ and $C_{(4)}^{2}$. There are no singular contributions from the $\phi$ field since the derivatives acting on it are not of a high enough order to produce a $\delta$-function.

We start with the calculation of the piece of (4.9) responsible for type A (Euler) anomaly. This requires evaluating the first two expressions to order $\epsilon$.

Since in $d=4 E_{(4)}$ is given by:

$$
E_{(4)}=\frac{1}{4} \epsilon^{i_{1} i_{2} j_{1} j_{2}} \epsilon^{i_{3} i_{4} j_{3} j_{4}} R_{i_{1} i_{2} i_{3} i_{4}} R_{j_{1} j_{2} j_{3} j_{4}}
$$

the singular curvature component (4.4) will single out the regular component $R_{a b c d}$ where the indices $a, b, c, d$ take the values 1,2. The singular components of the Einstein tensor using (4.5) will be in the 1,2 directions only, i.e.

$$
\bar{G}_{a b}=-4 \pi \epsilon \delta^{(2)}(z, \bar{z}) g^{z \bar{z}} g_{a b} .
$$


Using (4.13) and (4.14) we obtain from the first two terms in (4.9) a singular, effectively twodimensional piece $\bar{W}_{2}$ whose expression is:

$$
\bar{W}_{2}^{A}=16 \pi \epsilon a_{4} \int d^{4} x \delta^{(2)}(z, \bar{z}) \sqrt{\operatorname{det}\left(g_{a b}\right)}\left[g^{a b} \partial_{a} \phi \partial_{b} \phi+\frac{1}{2} \phi \epsilon^{a b} \epsilon^{c d} R_{a b c d}\right] .
$$

We remark that in (4.15) the two-dimensional $\epsilon$-symbols contain one inverse power of $\sqrt{\operatorname{det}\left(g_{a b}\right)}$.

The second term in (4.15) has an invariant meaning following from the fact that $g_{a b}$ and $R_{a b c d}$ at $z=\bar{z}=0$ are the induced metric and the pull back of the Riemann tensor, respectively. Then using the Gauss-Codazzi relation:

$$
R_{a b c d}^{(2)}=\partial_{a} X^{i} \partial_{b} X^{j} \partial_{c} X^{k} \partial_{d} X^{l} R_{i j k l}-g_{i j}\left(K_{a c}^{i} K_{b d}^{j}-K_{a d}^{i} K_{b c}^{j}\right)
$$

where $R^{(2)}$ is calculated with the induced metric and $K_{a b}^{i}$ is the second fundamental form, we can rewrite $\bar{W}_{2}$ as:

$$
\bar{W}_{2}^{A}=16 \pi \epsilon a_{4} \int d^{4} x \delta^{(2)}(z, \bar{z}) \sqrt{\operatorname{det}\left(g_{a b}\right)}\left[\phi R^{(2)}+g^{a b} \partial_{a} \phi \partial_{b} \phi+\phi \Delta\right]
$$

where $\Delta$ is the contribution of the second fundamental form given by:

$$
\Delta=g_{i j}\left[\operatorname{tr}\left(K^{i}\right) \operatorname{tr}\left(K^{j}\right)-\operatorname{tr}\left(K^{i} K^{j}\right)\right]
$$

the traces in (4.18) being taken with the induced metric.

The first two terms in (4.17) are the same as in the usual two-dimensional Wess-Zumino action (4.8) written in terms of the two-dimensional induced metric. Therefore they will produce the standard Polyakov anomaly $\sigma \sqrt{\operatorname{det}\left(g_{a b}\right)} R^{(2)}$. The additional term has, however, a nonvanishing Weyl variation following from the variation of the second fundamental form (3.11):

$$
\delta_{\sigma} \Delta=-2 \sigma \Delta-2 \partial_{i} \sigma \operatorname{tr}\left(K^{i}\right) .
$$

It follows that the total Weyl variation of $\bar{W}_{2}$ will be:

$$
\delta_{\sigma} \bar{W}_{2}^{A}=16 \pi \epsilon a_{4} \int d^{4} x \delta^{(2)}(z, \bar{z}) \sqrt{\operatorname{det}\left(g_{a b}\right)}\left[\sigma\left(R^{(2)}+\Delta\right)-2 \phi \partial_{i} \sigma \operatorname{tr}\left(K^{i}\right)\right] .
$$

We see therefore besides an addition $\Delta$ to the Polyakov anomaly a signal for nonlocality in the appearance of a term which still contains $\phi$ after the Weyl variation is taken. Indeed, if we eliminate $\phi$ through the equation of motion we obtain an expression for $\bar{W}_{2}$ proportional to:

$$
\int d^{4} x \delta^{(2)}(z, \bar{z}) \sqrt{\operatorname{det}\left(g_{a b}\right)}\left[R^{(2)}+\Delta\right] \frac{1}{\square^{(2)}}\left[R^{(2)}+\Delta\right]
$$

The Weyl variation of (4.21) contributed by the $\Delta$ terms does not cancel $\square^{(2)}$ in the denominator and it remains nonlocal. This is in contradiction with the analytic structure of a DNG contribution to the holographic action as we discussed it in Section 3.2.

We can try to make the "splitting" into the two-dimensional and four-dimensional contributions after the Weyl variation was taken, i.e. directly in the expression

$$
\delta_{\sigma} W_{4}=a_{4} \int d^{4} x \sqrt{\operatorname{det}(g)} \sigma E_{4} .
$$


From the expressions already used above we obtain:

$$
\begin{aligned}
\delta_{\sigma} W_{4}= & a_{4} \int d^{4} x \sqrt{\operatorname{det}(g)} E_{4}^{(\mathrm{reg})} \\
& +16 \pi \epsilon a_{4} \int d^{4} x \delta^{(2)}(z, \bar{z}) \sqrt{\operatorname{det}\left(g_{a b}\right)} \sigma\left[R^{(2)}+\Delta\right],
\end{aligned}
$$

where $E_{4}^{(\text {reg })}$ contains the contribution of the curvature components not having the $\delta$ functions. The second contribution is now by definition local. However if an anomaly is originating from an effective action it should obey the Wess-Zumino condition following from the (Abelian) algebra of Weyl transformations:

$$
\left(\delta_{2} \delta_{1}-\delta_{1} \delta_{2}\right) W=0
$$

where $\delta_{i}$ is a short hand for a variation with Weyl parameter $\sigma_{i}$ for $i=1,2$.

Using (4.19) we can verify directly that the second term in (4.23) does not satisfy the WessZumino condition so also this second way of "splitting" is not tenable. We remark that what prevents a consistent splitting is the presence of $\Delta$, related to a nonvanishing second fundamental form.

It is instructive to check under which conditions the four-dimensional Euler anomaly obeys the Wess-Zumino condition and why after the splitting the condition is not fulfilled anymore. The Weyl variation of the Euler density is given by:

$$
\delta_{\sigma} \sqrt{\operatorname{det}(g)} E_{4}=-8 \sqrt{\operatorname{det}(g)} G^{i j} \nabla_{i} \nabla_{j} \sigma
$$

where $G^{i j}$ is the Einstein tensor defined in (4.10). Using (4.25) in (4.22) in order to calculate the double variation we obtain:

$$
\left(\delta_{2} \delta_{1}-\delta_{1} \delta_{2}\right) W=-8 a_{4} \int d^{4} x \sqrt{\operatorname{det}(g)} G^{i j}\left[\sigma_{1} \nabla_{i} \nabla_{j} \sigma_{2}-\sigma_{2} \nabla_{i} \nabla_{j} \sigma_{1}\right]
$$

which is 0 after an integration by parts, provided:

$$
\nabla_{i} G^{i j}=0
$$

The Bianchi identity (4.27) is satisfied automatically for any metric. The metric we use has, however, a singular component (4.2) which produces Einstein tensor components which contain $\delta$-functions (4.14) and therefore the way (4.27) is satisfied is rather special. The $j=z$ component of the Bianchi identity (4.27) will contain also terms with $\delta$ functions. Their cancellation requires:

$$
g^{z \bar{z}} \partial_{\bar{z}} G_{z z}-g^{a b} \Gamma_{a z}^{c} G_{b c}=0 .
$$

where the most singular contribution to $G_{b c}$ is given by (4.14).

The component in the first term following from (4.2) is:

$$
\tilde{G}_{z z}=-\frac{1}{2} \partial_{z} g_{a b} \partial_{z}\left[\epsilon g_{z \bar{z}} \log (z \bar{z})\right] .
$$

After using (4.29) and (4.14) in (4.28) the identity is indeed satisfied however this required a term with an explicit $\delta$-function (4.14) canceling against (4.29) which produced a $\delta$-function only after being acted upon by a derivative. In the "splitting" process (4.14) is included in the two-dimensional piece while (4.29) in the four-dimensional one and the Wess-Zumino condition which required (4.27) is not anymore obeyed. A similar argument based on the inspection of (4.9) shows that the vanishing of the term containing the $\phi$ field in the Weyl variation, the necessary 
condition for the correct analyticity, is controlled by the same Bianchi identity (4.27). When the second fundamental form vanishes both $\Gamma_{a b}^{c}$ and $\tilde{G}_{z z}$ in (4.28) vanish separately and the Bianchi identity is satisfied trivially.

The mechanism discussed above which prevents a consistent "splitting" for the universal piece of the effective action responsible for the Euler trace anomaly could be rather general: the correct analyticity of the effective action probably requires various Bianchi identities which mix singular components of the curvatures (included in the two-dimensional piece) with components which become singular only after the application of derivatives.

We discuss now the second trace anomaly, the term in (4.9) with coefficient $c_{4}$. The fourdimensional Weyl tensor is given by:

$$
C_{i j k l}=R_{i j k l}-\frac{1}{2}\left[g_{i k} R_{j l}+g_{j l} R_{i k}-g_{j k} R_{i l}-g_{i l} R_{j k}\right]+\frac{1}{6}\left[g_{i k} g_{j l}-g_{j k} g_{i l}\right] R .
$$

The singular components of $C_{i j k l}$ are:

$$
\begin{aligned}
& \bar{C}_{z \bar{z} z \bar{z}}=-\frac{4 \pi \epsilon}{3} g_{z \bar{z}} \delta^{(2)}(z, \bar{z}), \quad \bar{C}_{z a \bar{z} b}=-\frac{2 \pi \epsilon}{3} g_{a b} \delta^{(2)}(z, \bar{z}), \\
& C_{a b c d}=\frac{4 \pi \epsilon}{3}\left[g_{a c} g_{b d}-g_{b c} g_{a d}\right] g^{z \bar{z}} \delta^{(2)}(z, \bar{z}) .
\end{aligned}
$$

Using the tracelessness of the Weyl tensor all the regular components can be expressed in terms of the pullback:

$$
g^{a b} C_{a z b \bar{z}}=-\frac{1}{2} g_{z \bar{z}} g^{a b} g^{c d} C_{a c b d}, \quad C_{z \bar{z} z \bar{z}}=-\frac{1}{2} g_{z \bar{z}}^{2} g^{a c} g^{b d} C_{a b c d} .
$$

Finally, using (4.31) and (4.32) in (4.9) we obtain for the last term the effectively twodimensional expression:

$$
\bar{W}_{2}^{B}=16 \pi \epsilon c_{4} \int d^{4} x \delta^{(2)}(z, \bar{z}) \sqrt{\operatorname{det}\left(g_{a b}\right)} \phi g^{a c} g^{b d} C_{a b c d} .
$$

Now a Weyl transformation which shifts $\phi$ transforms the Weyl tensor homogeneously and therefore the $\phi$ field is not present in the anomaly indicating that the "splitting" is consistent for this term in the effective action. Indeed, the anomaly is obtained by replacing $\phi$ in (4.33) by the Weyl transformation parameter $\sigma\left(x^{k}\right)$ and the Wess-Zumino condition is satisfied trivially. This is probably related to the fact that type $\mathrm{B}$ trace anomalies have a trivial descent, i.e. the consistency conditions do not require Bianchi identities. Eq. (4.33) has a consistent two-dimensional interpretation the invariant form of the anomaly following from (4.33) having the form:

$$
\delta_{\sigma} W_{2}=c t . \int d^{2} \tau \sqrt{\operatorname{det}(h)} \sigma h^{a c} h^{b d} C_{i j k l} \partial_{a} X^{i} \partial_{b} X^{j} \partial_{c} X^{k} \partial_{d} X^{l},
$$

where $h_{a b}$ is the induced metric. The holographic representation should produce therefore a Graham-Witten anomaly of the form (4.34).

In the special case when $a_{4}=0$ in order to integrate out the type B anomaly we need to add a Weyl invariant term to the Wess-Zumino action as discussed at the beginning of the section.

In summary, the analytic structure of the type A (Euler) Weyl anomaly term in the effective action of the CFT representing the EE is different than the one of its supposed holographic representation. This was obtained under two working assumptions, i.e. that the effective action could be used also for singular metrics and that a first order expansion in $\epsilon$ is safe. 


\section{The back reaction and the Graham-Witten anomalies}

The holographic realization as used in Section 3 involved smooth bulk metrics. Since in the CFT the singularity of the metric as reflected in Eq. (4.4) played an essential role we would like to examine if in the holographic realization such singular metrics could appear and if they may have an influence on the discrepancy discussed in the previous section. Of course the boundary value of the metric $g^{(0)}$ is smooth but the solution in the bulk can acquire singular components if the back reaction of the DNG component of the action is taken into account.

In Section 3 we have treated the dynamics of the bulk independently producing a solution $g_{i j}(x, \rho)$. The embedded surface evolved in this bulk background following the dynamics prescribed by the DNG action. In this section we will take back reaction of the hypersurface on the bulk into account, solve the coupled equations of motion and evaluate the $\mathcal{O}\left(\rho^{-1}\right)$ term of the onshell action. According to [13] this computes the Weyl anomaly, in addition to the contribution coming from the DNG piece.

The total action is

$$
S=\int_{\mathcal{M}} d \rho d^{d} x \sqrt{G}(R-2 \Lambda)+\int_{\Sigma} d \tau d^{k} y \sqrt{h} .
$$

The equation of motion for the metric can be cast in the form

$$
\sqrt{G}\left(\frac{1}{2}(R-2 \Lambda) G^{\mu \nu}-R^{\mu \nu}\right)=\Delta^{\mu \nu}
$$

with

$$
\Delta^{\mu \nu}=-\frac{1}{2} \int_{\Sigma} \sqrt{h} h^{\mu v} \delta^{(d)}(x-X(\tau)) \delta(\rho-\tau) .
$$

Inserting its trace into the action results in

$$
S=2 d \int_{\mathcal{M}} \sqrt{G}+\frac{d-k-2}{d-1} \int_{\Sigma} \sqrt{h}
$$

where the second term vanishes if $\operatorname{codim}(\Sigma)=2$, which is the case of interest where $d=4$ and $k=2$. However, the DNG piece of the action will feed back, through the equations of motion, into the coefficients $g_{i j}^{(n)}$.

Using the FG expansion (3.7) one finds the following expression for the on-shell action at $\mathcal{O}\left(\rho^{-1}\right)[13]$

$$
2 \sqrt{\operatorname{det} g^{(0)}}\left(\operatorname{tr} \stackrel{(2)}{g}-\frac{1}{2} \operatorname{tr}\left(\stackrel{(1)}{g}^{2}\right)+\frac{1}{4}(\operatorname{tr} \stackrel{(1)}{g})^{2}\right) .
$$

For $g^{(1)}$ one finds, by solving the $(i j)$-component of (5.2) at leading non-trivial order in its $\rho$ expansion

$$
\stackrel{(1)}{g}_{i j}=\frac{1}{2}\left(\stackrel{(0)}{R}_{i j}-\frac{1}{6} \stackrel{(0)}{R} \stackrel{(0)}{g}_{i j}\right)+\delta \stackrel{(1)}{g}_{i j},
$$

where

$$
\delta_{g_{i j}}^{(1)}=-\frac{1}{4 \sqrt{g^{(0)}}} \int d^{2} y \sqrt{\stackrel{(0)}{h}}\left(\stackrel{(0)}{h}_{i j}-\frac{2}{3} \stackrel{(0)}{g}_{i j}\right) \delta^{(4)}(x-\stackrel{(0)}{X}(y))
$$


and

$$
\stackrel{(0)}{h}_{i j}=\stackrel{(0)}{g}{ }_{i k} \stackrel{(0)}{g}_{j l} \stackrel{(0)}{h^{a b}} \partial_{a} \stackrel{(0)}{X}^{k} \partial_{b} \stackrel{(0)}{X}^{l} .
$$

Note that $\delta g^{(1)}$ is Weyl invariant and $g^{(1)}$ is no longer universal. Consistency with PBH and dimensional arguments restrict the most general nonuniversal addition to $g^{(1)}$, which would result for general bulk and hypersurface action to the above form, but with arbitrary coefficients for $h^{(0)}$ and $g^{(0)}$ in (5.7).

To find $\operatorname{tr}\left(g^{(2)}\right)$ it suffices to solve the $(\rho \rho)$-component of (5.2) at lowest non-trivial order:

$$
\operatorname{tr}(\stackrel{(2)}{g})=\frac{1}{4} \operatorname{tr}(\stackrel{(1)}{g} 2)-\frac{1}{32} \frac{1}{\sqrt{g^{(1)}}} \int d^{2} y \sqrt{{ }_{h}^{(0)}} K^{i} K^{j_{g}^{(0)}} \delta_{i j}^{(4)}(x-\stackrel{(0)}{X}) .
$$

The expressions (5.6) and (5.9) represent singular contributions to the bulk metric solution. Using the singular contributions to linear order in the $\delta$-function we will find the contributions to the Graham-Witten anomaly. Quadratic and higher order terms in the $\delta$-functions require a regularization producing local counterterms which do not influence the anomalies.

We find for (5.5) for the case $d=4, k=2$

$$
\begin{aligned}
& \left.\frac{1}{2} \sqrt{{ }_{(0)}^{g}}\left((\operatorname{tr} \stackrel{(1)}{g})^{2}-\operatorname{tr}(\stackrel{(1)}{g})\right)\right)\left.\right|_{\text {universal }} \\
& \left.\quad-\frac{1}{16} \int d^{2} y \sqrt{\stackrel{(0)}{h}\left(K^{i} K^{j^{(0)}}\right.}{ }_{i j}-4 h^{a}{ }^{(\stackrel{(1)}{g}}{ }_{a b}\right) \delta^{(4)}(x-\stackrel{(0)}{X}),
\end{aligned}
$$

where the universal $g^{(1)}$ was given in (3.10). Again there was a crucial cancellation, related to the one observed above, for $\operatorname{codim}(\Sigma)=2$.

Compare (5.10) to (3.35): we have shown that for the simplest bulk and hypersurface actions, taking into account the back-reaction leads to the same GW anomaly for the total action (5.1).

The above result has a simple explanation which will allow us to generalize the result for arbitrary bulk actions. The contributions to the bulk metric specified above once inserted in the bulk action to linear order in the $\delta$-function produce a term localized on the submanifold. Moreover this term has the same symmetries as the DNG action. Therefore we can use the procedure discussed in Section 3.2. The additional Graham-Witten anomaly is given by an expression analogous to (3.31) the DNG integrand being replaced by the term of the bulk action specified above. We will need therefore just the expansion to order $\tau^{2}$ (order $\rho^{2}$ in our gauge) of the integrand in the first term in (5.1). Remembering that $g^{(2)}$ does not appear in the expansion we get the following terms (the curvatures are computed with $g^{(0)}$ ):

$$
\operatorname{tr}\left(\stackrel{(1)}{g}^{2}\right)-(\operatorname{tr} \stackrel{(1)}{g})^{2}-\stackrel{(1)}{g}_{i j} R^{i j}+\frac{1}{2} R \operatorname{tr} \stackrel{(1)}{g}
$$

In the expression (5.11) we left out terms in which derivatives act on $g^{(1)}$. We will discuss them in the general setting.

Now, using (5.6) in (5.11) it is easy to verify that all the terms linear in $\delta g^{(1)}$ vanish without any need to specify the exact coefficients in $\delta g^{(1)}$. What is the reason for this vanishing? As we discussed in Section 3.2 an expression obtained by (3.31) satisfies automatically the WessZumino condition. Independently of the exact form of the bulk action for dimensional reasons the only expressions which could appear in (5.11) linear in $\delta g^{(1)}$ are $R_{a b}$-the pullback of the Ricci curvature or $R$ - the bulk scalar curvature restricted to the submanifold. Indeed they do appear in individual terms in (5.11). However once they are multiplied with the Weyl parameter 
$\sigma$ it is easy to check that they do not fulfill the Wess-Zumino condition and therefore they must cancel in the full expression.

Finally we return to the derivative terms left out above. Again by a dimensional argument verified explicitly for the aforementioned terms these contributions have the form $\square \sigma$ or $K^{i} \partial_{i} \sigma$, restricted to the submanifold. These expressions do satisfy the Wess-Zumino condition but they are cohomologically trivial being the variations of local expressions as we discussed in Section 3.2 .

In conclusion, for an arbitrary bulk action in $d=5$ and an arbitrary three-dimensional DNG action the Graham-Witten anomalies remain unchanged after the back reaction on the bulk metric is included. This is a consequence of the fact that the Graham-Witten anomalies classified in Section 3.2 cannot originate from the $g^{(1)}$ back reaction term the only one available in $d=5 .^{5}$

\section{Discussion}

The holographic representation of the entanglement entropy by adding to the bulk action a DNG type action is problematic for the reasons discussed in the previous sections. The analytic structure of certain terms in the effective action in the CFT is different than the one obtained through the aforementioned holographic mapping. The difference in the analytic structure leads to quantitative discrepancies even in the simplest case. We illustrate this fact by a calculation of a certain term in the entanglement entropy when our CFT is formulated in a flat metric background and the two regions in space are the exterior and interior of a sphere of radius $\bar{r}$.

The entanglement entropy depends on $\bar{r}$ through terms containing an ultraviolet cut-off. In addition there is the possibility of a universal logarithmic dependence. To put in evidence this term one considers constants appearing in:

$$
\bar{r} \frac{d}{d \bar{r}} S .
$$

In this form it is clear that (6.1) is related to the trace anomaly, a change in scale of $\bar{r}$ being produced by a joint constant Weyl rescaling and a rescaling diffeomorphism. A constant Weyl transformation produces a nonzero result if the anomaly is type B and likewise for the type A Euler density provided that the manifold has a nonzero Euler characteristic, which is the case for a sphere.

On the CFT side the calculation is straightforward: besides the bulk part which is identically 0 for a flat metric involving to order $\epsilon$ only $R \ldots$ and $\tilde{R} \ldots$ there are the singular contributions (4.23) and (4.33):

$$
\bar{r} \frac{d}{d r} S=16 \pi \int d^{4} x \delta^{(2)}(z, \bar{z}) \sqrt{\operatorname{det}\left(g_{a b}\right)}\left[a_{4}\left(R^{(2)}+\Delta\right)+c_{4} g^{a c} g^{b d} C_{a b c d}\right] .
$$

Now $C_{a b c d}$ vanishes for a flat metric and so does the first term in (6.2) since it really represents $R_{a b c d}$. The vanishing of $R_{a b c d}$ explains the relation:

$$
R^{(2)}=-\Delta
$$

\footnotetext{
5 Exactly the same argument leads to the identical conclusion for arbitrary codimension. This is so because for $k=2$ for dimensional reasons only $\delta g^{(1)}$ of the form (5.7), but with arbitrary coefficients for $h^{(0)}$ and $g^{(0)}$, can contribute. We expect that one can also relax the condition $k=2$, in other words, that the back reaction never changes the GW anomalies. This was observed and explained in [21] for conventional matter couplings to bulk gravity. We thank K. Skenderis for illuminating email exchange on this issue.
} 
where $\Delta$ is given by (4.18) which can be verified directly for a sphere embedded in a flat metric. As a result the constant which could have appeared in (6.1) vanishes.

On the holographic side the contribution to (6.1) can come from one of the Graham-Witten anomalies we studied in Section 3. The expressions for the two type B anomalies, the pull back of $C_{i j k l}$ and $g_{i j}\left[\frac{1}{2} \operatorname{tr}\left(K^{i}\right) \operatorname{tr}\left(K^{j}\right)-\operatorname{tr}\left(K^{i} K^{j}\right)\right]$ vanish for the sphere as can be checked explicitly. On the other hand the type A anomaly $R^{(2)}$ does not vanish and integrates to the Euler number of the sphere. As we discussed in Section 3 this anomaly does not vanish for the DNG action and it is there even for very general forms of the three-dimensional action having the symmetries of the DNG action. By "fine tuning" the additional terms the coefficient of $R^{(2)}$ can presumably be made to vanish but the presence of these terms in a systematic large $N$ expansion is not justified. We have therefore a clear contradiction between the field theoretical calculation and its proposed holographic representation.

Of course the DNG action can represent holographically other, "generalized Wilson loop type" observables in the CFT as discussed in [7-9]. As shown in the aforementioned references these observables have Graham-Witten anomalies completely compatible with the ones produced by the holographic representation.

We are faced therefore with the problem of producing a holographic representation of the entanglement entropy which is compatible with the field theoretical constraints. An obvious guess would be simply a bulk gravitational action whose classical solution matches at the boundary the singular metric studied in Section 2.

As far as the terms in the action producing the trace anomalies are concerned this proposal seems to be valid, though in a rather tautological way: as we discussed in Section 3 the calculation of the trace anomalies does not require the solution of the equations of motion but just the evaluation of some boundary terms for the boundary metric. Therefore the holographic calculation is bound to reproduce the results of Section 4.

For other terms of the action the solution of the equations of motion with singular boundary conditions would be needed and it is far from obvious that such a calculation can be controlled.

A related question is the appearance and interpretation of nonuniversal contributions in the effective action of the CFT representing the EE. As a concrete example we consider again the EE for a sphere embedded in a flat metric. On general grounds [1] one expects for the EE a leading dependence proportional to $\bar{r}^{2}$ multiplied by an appropriate scale. Are such terms obtainable in the CFT from Weyl invariant contributions to the effective action which however being more singular than the terms we considered require an additional regularization for the singular metric or are simply, nonuniversal subtractions (boundary terms) like in the holographic representation?

These and other questions related to the holographic representation of the EE are presently under study.

\section{Acknowledgements}

Very useful discussions with M. Bañados, N. Boulanger, D. Giulini, D. Kutasov, H. Neuberger, S. Shankaranarayanan and H. Shimada are gratefully acknowledged.

\section{Appendix A. Derivation of the universal type A anomaly coefficient}

In [10] it was shown that for any gravitational action (3.1) with has $A d S_{2 n+1}$ as a solution to the equations of motion, the coefficient $a$ of the unique type A anomaly of dual CFT is 


$$
a_{n}=\frac{b_{0}}{2^{2 n}(n !)^{2}},
$$

where $b_{0}=f(A d S)$. In [10] this was derived by looking at a conformally flat metric $g^{(0)}$ and solving the PBH-transformation equation for $b_{n}$. Here we will present an alternative derivation which uses the ideas of [22]. There it was observed that while the type B Weyl anomalies have a trivial descent, the unique (in any even dimensions) type A anomaly has a non-trivial descent. ${ }^{6}$ These features might, in fact, serve as the defining distinction between the two classes of anomalies, which can also be applied to the hypersurface anomalies discussed in Section 3.

We begin with a review of the results of [22]. Define

$$
\begin{aligned}
\mathcal{O}_{12 \ldots p+1}^{j_{1} \ldots j_{p}}= & \frac{4^{p} n !}{2^{n}(n-p) !} \sqrt{g} g_{i_{1} k_{1}} \ldots g_{i_{p} k_{p}} \epsilon^{i_{1} j_{1} \ldots i_{n} j_{n}} \epsilon^{k_{1} l_{1} \ldots k_{n} l_{n}} \\
& \times R_{i_{p+1} j_{p+1} k_{p+1} l_{p+1}} \cdots R_{i_{n} j_{n} k_{n} l_{n}} \sigma_{[1} \partial_{l_{1}} \sigma_{2} \ldots \partial_{l_{p}} \sigma_{p+1]},
\end{aligned}
$$

where the antisymmetrization is over the indices of $\sigma$. In particular

$$
\mathcal{O}_{1}=\sigma_{1} \sqrt{g} E_{2 n}
$$

with

$$
E_{2 n}=\frac{1}{2^{n}} \epsilon^{i_{1} j_{1} \ldots i_{n} j_{n}} \epsilon^{k_{1} l_{1} \ldots k_{n} l_{n}} R_{i_{1} j_{1} k_{1} l_{1}} \cdots R_{i_{n} j_{n} k_{n} l_{n}}
$$

the $d$-dimensional Euler density. The normalization is such that $E_{2 n}=R^{n}+\cdots \cdot \mathcal{O}_{1}$ is at the top of the descent which is

$$
\delta_{[p+1} \mathcal{O}_{1 \ldots p]}^{j_{1} \ldots j_{p-1}}=\partial_{p} \mathcal{O}_{1 \ldots p+1}^{j_{1} \ldots j_{p}}
$$

and

$$
\mathcal{O}_{12 \ldots n+1}^{j_{1} \ldots j_{n}}=2^{n}(n !)^{2} \sqrt{g} \sigma_{[1} \nabla^{j_{1}} \sigma_{2} \cdots \nabla^{j_{n}} \sigma_{n+1]}
$$

is at the bottom. In deriving (A.5) we need the Weyl variation of the Riemann tensor

$$
\delta R_{i j k l}=2 \sigma R_{i j k l}+g_{i k} \nabla_{j} \nabla_{l} \sigma+g_{j l} \nabla_{i} \nabla_{k} \sigma-g_{i l} \nabla_{j} \nabla_{k} \sigma-g_{j k} \nabla_{i} \nabla_{l} \sigma .
$$

The holographic version of the descent starts with the $(d+1)$-dimensional ' $\mathrm{CS}$-form' $\mathcal{O}=$ $\sqrt{G} f(R)$. Under PBH

$$
\delta_{1} \mathcal{O}=\partial_{\mu}\left(\xi_{1}^{\mu} \mathcal{O}\right) \equiv \partial_{\mu} \mathcal{O}_{1}^{\mu} .
$$

If we define

$$
\mathcal{O}_{1 \ldots p}^{\mu_{1} \ldots \mu_{p}}=\xi_{[1}^{\mu_{1}} \cdots \xi_{p]}^{\mu_{p}} \mathcal{O}
$$

we can show, using the group property (3.4)

$$
\delta_{p+1} \mathcal{O}_{1 \ldots p}^{\mu_{1} \ldots \mu_{p}}=\partial_{\mu_{p+1}} \mathcal{O}_{1 \ldots p+1}^{\mu_{1} \ldots \mu_{p+1}} .
$$

Using (3.8) for the $\rho$-expansion of $\mathcal{O}$ and $\xi^{\rho}=2 \sigma \rho, \xi^{i}=\frac{1}{2} \rho g_{(0)}^{i j} \partial_{j} \sigma+\mathcal{O}\left(\rho^{2}\right)$ we find

$$
\mathcal{O}_{1 \ldots n+1}^{\rho j_{1} \ldots j_{n}}=\frac{1}{2^{n}} \sqrt{g} b_{0} \sigma_{[1} \nabla^{j_{1}} \sigma_{2} \cdots \nabla \sigma_{n+1]}^{j_{n}} .
$$

Comparing this with (A.6) we conclude that the holographic type A Weyl anomaly in $d=2 n$ dimensions is $a_{n} E_{2 n}$ with $a_{n}$ as in (A.1).

\footnotetext{
6 The descent of cohomologically trivial contributions stops after the second step.
} 


\section{References}

[1] Some early references on the entanglement entropy are L. Bombelli, R.K. Koul, J.H. Lee, R.D. Sorkin, A quantum source of entropy for black holes, Phys. Rev. D 34 (1986) 373;

M. Srednicki, Entropy and area, Phys. Rev. Lett. 71 (1993) 666, hep-th/9303048;

C. Holzhey, F. Larsen, F. Wilczek, Geometric and renormalized entropy in conformal field theory, Nucl. Phys. B 424 (1994) 443, hep-th/9403108.

[2] P. Calabrese, J.L. Cardy, Entanglement entropy and quantum field theory, J. Stat. Mech. 0406 (2004) P002, hepth/0405152;

P. Calabrese, J.L. Cardy, Entanglement entropy and quantum field theory: A non-technical introduction, Int. J. Quantum Inf. 4 (2006) 429, quant-ph/0505193.

[3] S. Ryu, T. Takayanagi, Holographic derivation of entanglement entropy from AdS/CFT, Phys. Rev. Lett. 96 (2006) 181602, hep-th/0603001;

S. Ryu, T. Takayanagi, Aspects of holographic entanglement entropy, JHEP 0608 (2006) 045, hep-th/0605073.

[4] D.V. Fursaev, Proof of the holographic formula for entanglement entropy, JHEP 0609 (2006) 018, hep-th/0606184.

[5] I.R. Klebanov, D. Kutasov, A. Murugan, Entanglement as a probe of confinement, arXiv: 0709.2140 [hep-th].

[6] T. Nishioka, T. Takayanagi, AdS bubbles, entropy and closed string tachyons, JHEP 0701 (2007) 090, hep-th/ 0611035.

[7] M. Henningson, K. Skenderis, Weyl anomaly for Wilson surfaces, JHEP 9906 (1999) 012, hep-th/9905163.

[8] A. Gustavsson, Conformal anomaly of Wilson surface observables: A field theoretical computation, JHEP 0407 (2004) 074, hep-th/0404150;

A. Gustavsson, On the Weyl anomaly of Wilson surfaces, JHEP 0312 (2003) 059, hep-th/0310037.

[9] V. Asnin, Analyticity properties of Graham-Witten anomalies, arXiv: 0801.1469 [hep-th].

[10] C. Imbimbo, A. Schwimmer, S. Theisen, S. Yankielowicz, Diffeomorphisms and holographic anomalies, Class. Quantum Grav. 17 (2000) 1129, hep-th/9910267.

[11] E. Witten, Anti-de Sitter space and holography, Adv. Theor. Math. Phys. 2 (1998) 253, hep-th/9802150.

[12] C. Fefferman, R. Graham, Conformal Invariants, Astèrisque, Hors Série, 1995, p. 95.

[13] M. Henningson, K. Skenderis, The holographic Weyl anomaly, JHEP 9807 (1998) 023, hep-th/9806087.

[14] A.D. Rendall, Asymptotics of solutions of the Einstein equations with positive cosmological constant, Ann. Henri Poincare 5 (2004) 1041, gr-qc/0312020.

[15] A. Schwimmer, S. Theisen, Universal features of holographic anomalies, JHEP 0310 (2003) 001, hep-th/0309064.

[16] I. Papadimitriou, K. Skenderis, Thermodynamics of asymptotically locally AdS spacetimes, JHEP 0508 (2005) 004, hep-th/0505190.

[17] C.R. Graham, E. Witten, Conformal anomaly of submanifold observables in AdS/CFT correspondence, Nucl. Phys. B 546 (1999) 52, hep-th/9901021.

[18] D.V. Fursaev, S.N. Solodukhin, On the description of the Riemannian geometry in the presence of conical defects, Phys. Rev. D 54 (1995) 2133, hep-th/9501127.

[19] J. Wess, B. Zumino, Consequences of anomalous Ward identities, Phys. Lett. B 37 (1971) 95.

[20] A. Cappelli, A. Coste, On the stress tensor of conformal field theories in higher dimensions, Nucl. Phys. B 314 (1989) 707;

E.S. Fradkin, A.A. Tseytlin, Conformal anomaly in Weyl theory and anomaly free superconformal theories, Phys. Lett. B 134 (1984) 187.

[21] M. Bianchi, D.Z. Freedman, K. Skenderis, How to go with an RG flow, JHEP 0108 (2001) 041, hep-th/0105276; M. Bianchi, D.Z. Freedman, K. Skenderis, Holographic renormalization, Nucl. Phys. B 631 (2002) 159, hepth/0112119.

[22] N. Boulanger, General solutions of the Wess-Zumino consistency condition for the Weyl anomalies, JHEP 0707 (2007) 069, arXiv: 0704.2472 [hep-th];

N. Boulanger, Algebraic classification of Weyl anomalies in arbitrary dimensions, Phys. Rev. Lett. 98 (2007) 261302, arXiv: 0706.0340 [hep-th]. 